\title{
Radio and X-ray Study of SNRs in the Magellanic Clouds
}

\author{
M.D. Filipović ${ }^{1,2,3}$, W. Pietsch ${ }^{1}$, G.L. White ${ }^{2}$, F. Haberl ${ }^{1}$, \\ L. Staveley-Smith ${ }^{3}$, P.A. Jones ${ }^{2}$, R.F. Haynes ${ }^{3}$ and M. Sasaki ${ }^{1}$ \\ 1 Max-Planck-Institut für extraterrestrische Physik, Garching, Germany \\ 2 University of Western Sydney, Nepean, NSW 2747, Australia \\ 3 Australia Telescope National Facility, CSIRO, NSW 2121, Australia
}

\begin{abstract}
We present our high-resolution radio-continuum and X-ray study of supernova remnants (SNRs) in the Magellanic Clouds (MCs). These investigations are based on Australia Telescope Compact Array (ATCA) radio-continuum and ROSAT X-ray observations. Our main aim is to study a complete sample of the MC SNRs and $\mathrm{H}$ II regions.
\end{abstract}

\section{Introduction}

Statistical studies of objects such as SNRs in our Galaxy, despite their large number $(\sim 220)$, have problems due to incompleteness and uncertain distances. Objects in the MCs have approximately the same distance and foreground absorption. However, they are still close enough to allow detailed spatially resolved investigations. The MCs contain all types of SNRs and $\mathrm{H}$ II regions in various stages of evolution. Also, the MCs fortunately lie in a direction well out of the Galactic plane and the foreground densities of dust, gas and stars are small. SNRs in other galaxies such as M31 or M33 appear about ten times smaller and one hundred times fainter than SNRs in the MCs.

From our low-resolution comparison between the Parkes radio and ROSAT All-Sky survey (RASS), we found that the majority of sources in common are SNRs (60 per cent) and background objects (30 per cent). A source-intensity comparison of radio and X-ray source flux densities shows very little correlation, but we note that the strongest SNRs at both frequencies are young SNRs from Population I (Filipović et al. 1998a).

About 80 SNRs and SNR candidates in the MCs have been identified in our recent studies (Filipović et al. 1998b). Of these, there are 23 SNR candidates in the LMC and 6 in the SMC which need to be confirmed as SNRs.

\section{The ATCA Observations of SNRs in the MCs}

The ATCA mosaic observations of the SMC were taken in October 1992. The baseline of $375 \mathrm{~m}$ was used at frequencies of 1.42 and $2.37 \mathrm{GHz}$ with angular resolution of $\sim 90^{\prime \prime}$ and $45^{\prime \prime}$, respectively. The total of 320 pointings covered an area of $\sim 20$ square degrees (Staveley-Smith et al. 1997). More details about the LMC mosaic observations can be found in Kim et al. (1998). The SMC mosaic 

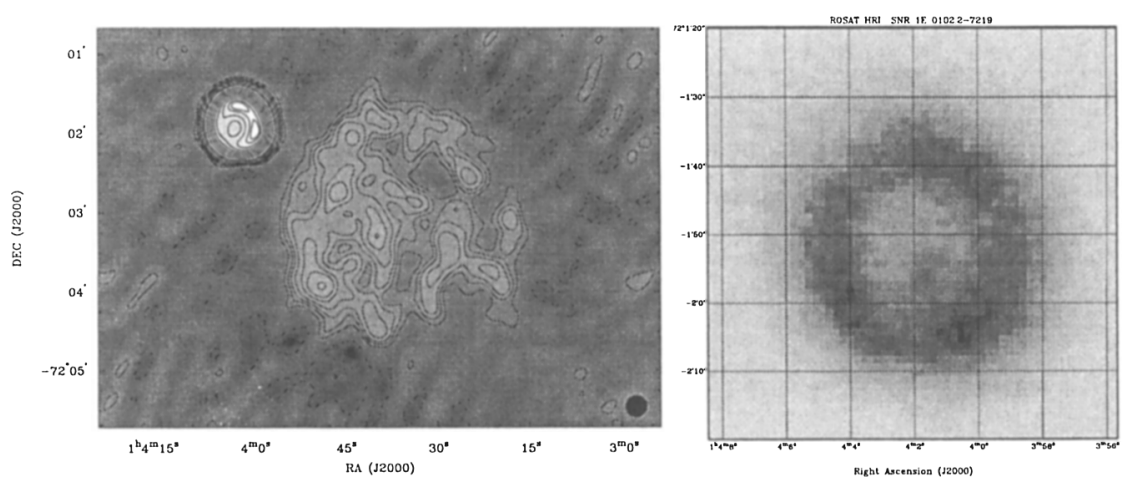

Figure 1. The snapshot image of the SMC region N 76 (Fig. 1a - left) at $8.64 \mathrm{GHz}$. The r.m.s. noise $(1 \sigma)$ of the image is $0.15 \mathrm{mJy}$ and the observed beam size is $15^{\prime \prime}$ (lower right corner). The resolved source in the north-east is SNR 1E 0102.2-7219 and a more detailed radiocontinuum study can be found in Amy \& Ball (1993). The extended superbubble in the center of Fig. 1a is $\mathrm{H}$ II region N 76A. Contours are: $-3 \sigma, 3 \sigma, 5 \sigma, 7 \sigma, 10 \sigma, 15 \sigma, 20 \sigma, 30 \sigma, 50 \sigma, 70 \sigma$ and $95 \sigma$. Fig. 1b (right) shows a high-resolution ROSAT HRI image of the SNR 1E 0102.2-7219 which contains two merged observations with a total exposure time of $36 \mathrm{ks}$.

images at both frequencies are "corrected" for missing short spacings by adding Parkes survey data. Over seven hundred sources were found in these surveys (Filipović et al. in prep.).

As a continuation of the Parkes radio-continuum investigations of sources intrinsic to the MCs and as an addition to the mosaic surveys, we used the ATCA in snapshot mode at $\nu=4.79 / 8.64 \mathrm{GHz}$ with the $375 \mathrm{~m}$ configuration. These observations, together with the ATCA mosaic surveys at $1.42 / 2.37 \mathrm{GHz}$ and the MOST survey at $843 \mathrm{MHz}$, will give us wide frequency coverage with sufficient resolution for detailed studies.

\section{The ROSAT X-ray Observations of the MCs}

The ROSAT archive contains over 240 PSPC and over 650 HRI pointing observations in the direction of the MCs. These observations were carried out with the ROSAT detectors (energy range 0.1-2.4 keV) from October 1991 until June 1998. A catalogue of more than 500 RASS X-ray sources towards the LMC is given in Pietsch et al. (in prep.). Kahabka et al. (1998) used part of the ROSAT PSPC observations towards the SMC and detected 248 objects which were included in the first version of the SMC catalogue of point and moderately extended soft X-ray sources. Two new updated X-ray catalogues (based on PSPC observations) of sources in the LMC (Haberl et al. in prep.) and the SMC (Filipović et al. in prep) revealed over 750 and $520 \mathrm{X}$-ray sources in the fields of the LMC and the SMC, respectively. 
SMC SNR 1E 0102.2-7219

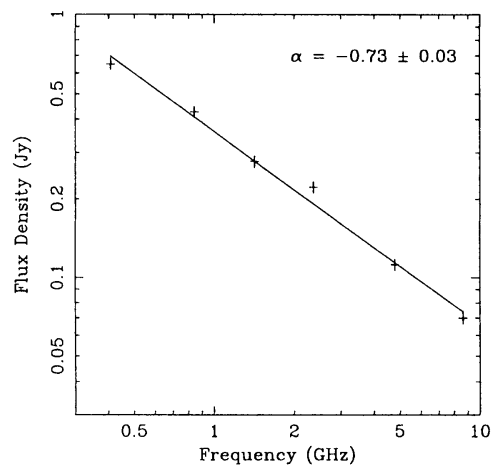

SMC N 76A (DEM S123)

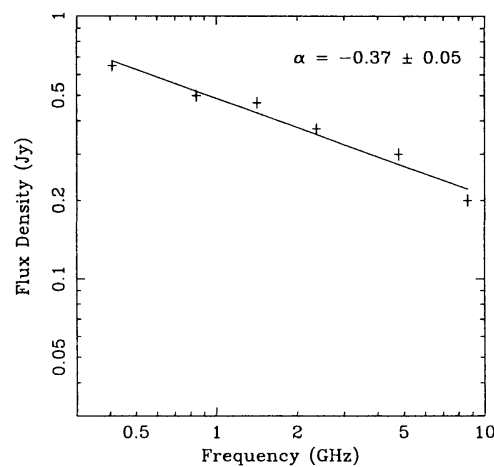

Figure 2. The radio-continuum spectrum of SNR 1E0102.2-7219 (Fig. 2a - left) and $\mathrm{H}$ II region N76A (Fig. 2b - right) in the SMC. The spectral index $\alpha$ is defined by the relation $\mathrm{S}_{\nu} \sim \nu^{\alpha}$, where $\mathrm{S}_{\nu}$ is the integrated flux density and $\nu$ is frequency. The integrated flux densities were estimated from our mosaic surveys $(1.42$ and $2.37 \mathrm{GHz})$, snapshot surveys $(4.79$ and $8.64 \mathrm{GHz})$ and from the MOST survey at $408 / 843 \mathrm{MHz}$ (Amy \& Ball 1993).

\section{Results}

In Figs. $1 \mathrm{a}$ and $1 \mathrm{~b}$ we present images from our study of the $\mathrm{N} 76$ region in the SMC. The image in Fig. 1a is taken with the ATCA in snapshot mode at $\nu=8.64 \mathrm{GHz}$ (beam size $\left.=15^{\prime \prime}\right)$. N 76 (DEM S123; SMC B0102-7218) is located in the northern part of the SMC and consists of a large H II superbubble (southwest) and SNR 1E0102.2-7219 (north-east) (Amy \& Ball 1993). In Fig. 1b we show the ROSAT HRI image of the SNR 1E0102.2-7219. Here, we can clearly see a ring-like structure typical for the oxygen-rich class of SNRs. With a radio spectral index of $\alpha=-0.73 \pm 0.03$ (Fig. 2a) we confirm this source as a SNR. Also, we note an "anti-correlation" between radio-continuum and X-ray emission from this SNR. This may be a typical characteristic for this class of SNRs. Some $\sim 3^{\prime}$ south-west from the SNR is the source N 76A which has been previously classified as an $\mathrm{H}$ II region. No X-ray emission can be detected from this superbubble and we estimate the radio spectral index to be $\alpha=-0.37 \pm 0.05$ (Fig. 2b) which is rather steep for $\mathrm{H}$ II regions.

A similar object to the SMC H II region $\mathrm{N} 76 \mathrm{~A}$ can be found in the LMC as the superbubble 30 Doradus $\mathrm{C}$ (also known as SNR 0536-69.2). Both objects are similar in size and spectra (30 Dor $\mathrm{C}-\alpha=-0.42 \pm 0.07$ ). However, the $\mathrm{SNR} /$ superbubble 30 Dor $\mathrm{C}$ has extended X-ray emission (Fig. $3 \mathrm{~b}$ ) which is not the case in SMC N 76A. Also, we note that there is a brightness displacement between radio and $\mathrm{X}$-ray emission which is not due to the positional uncertainties of either X-ray or radio observations. This displacement was initially discovered in Einstein surveys (Mathewson et al. 1985) and we believe that it represents different physical mechanisms being triggered inside this SNR/superbubble. 


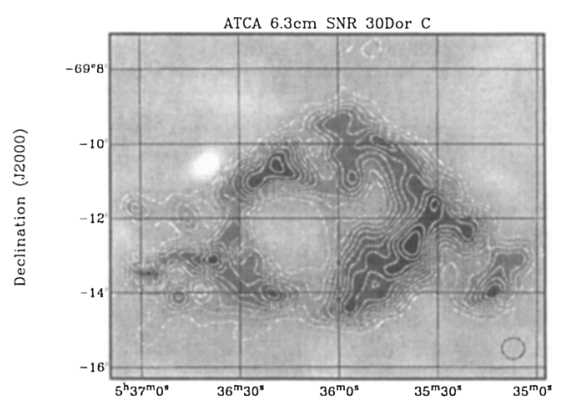

Right Ascension (J2000)

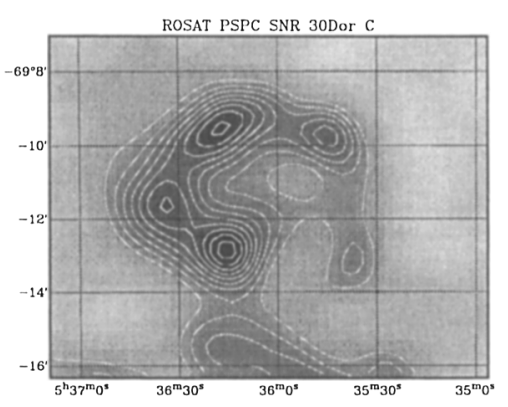

Right Ascension (J2000)

Figure 3. The radio-continuum $(4.79 \mathrm{GHz})$ and ROSAT PSPC X-ray images of the superbubble 30 Dor C. The r.m.s. noise $(1 \sigma)$ of the radio-continuum image (left) is $0.5 \mathrm{mJy}$ and the observed beam size is $30^{\prime \prime}$ (lower right corner). The noise on the X-ray image (right) is $0.7 \mathrm{cts}$. The positional displacement and anti-correlation between radio and X-ray emission is due to a different production mechanisms triggered in this SNR/superbubble.

\section{Future Work}

Individual SNRs will be studied in more detail. Some of their characteristics may be related to peculiar properties of the interstellar medium around the explosion site and/or different precursor stars. Theoretical models indicate that changes in most properties of the surrounding medium can have a significant effect upon the evolution of an SNR and its emission. However, the collective properties of the SNRs in the MCs studied to date are surprisingly consistent and quite similar to the population of Galactic SNRs (Dickel \& Milne 1994), despite major differences between these galaxies.

The luminosity-diameter distribution will be used to study the evolution of SNRs in a statistical sense. Also a comparison with the optical and IR surveys such as $\mathrm{H} \alpha,[\mathrm{SII}],[\mathrm{OIII}]$, IRAS and DENIS are planned as well.

\section{References}

Amy, S.W., Ball, L. 1993, ApJ, 411, 761

Dickel, J.R., Milne, D.K. 1994, Proc. ASA, 11, 99

Filipović, M.D., Pietsch, W., Haynes, R.F., et al. 1998a, A\&AS, 127, 119

Filipović, M.D., Haynes, R.F., White, G.L., Jones, P.A. 1998b, A\&AS, 130, 421 Kahabka, P., Pietsch, W., Filipović, M.D., Haberl, F. 1998, A\&AS, submitted Kim, S., Staveley-Smith, L., Dopita, M.A., et al. 1998, ApJ, 503, 674 Mathewson, D.S., Ford, V.L., Tuohy, I.R., et al. 1985, ApJS, 58, 197 Staveley-Smith, L., Sault, R.J., Hatzidimitriou, D., et al. 1997, MNRAS, 289, 225 